\title{
Model-Based Estimation of Decarburization Rate and Carbon Content in the Basic Oxygen Steelmaking Process
}

\author{
Andreas Johansson $^{* \dagger}$, Alexander Medvedev ${ }^{\dagger}$, and Daniel Widlund ${ }^{\ddagger}$ \\ † Control Engineering Group, Luleå University of Technology, SE-971 87 Luleå, Sweden \\ $\ddagger$ SSAB Oxelösund AB, SE-613 80 Oxelösund, Sweden \\ *e-Mail Andreas.Johansson@sm.luth.se Phone +46920723 34 Fax +46920915 58
}

\begin{abstract}
The problem of metal analysis estimation in real time for the basic oxygen steelmaking process is considered. A nonlinear observer is developed and shown to be locally asymptotically stable. In experiments with real data, it provides accurate estimates of the carbon content in the converter.
\end{abstract}

\section{INTRODUCTION}

The top blown basic oxygen method has been used for 50 years and is today the predominant process for steelmaking. In 1995 it accounted for nearly $60 \%$ of the world total crude steel production of almost 750 million metric tons.

The aim of the process is to reduce the contents of carbon and silicon and other components in the hot metal from the blast furnace. The main quality measure of the final product is the carbon content. On the one hand, if the preset upper limit is exceeded, then the process must be repeated, which is very time consuming.

On the other hand, there is a considerable extra cost in terms of consumption of resources, such as oxygen, energy, and production time, when the final carbon content is made unnecessarily low.

Being able to accurately estimate the metal analysis, especially the content of carbon, is thus important for ensuring the quality of the steel, minimizing the consumption of resources and to avoid re-blowing. The use of an analytical process model is a promising way of achieving this.

There exist a few commercial systems for on-line estimation of metal analysis, e.g. MEFCON [1], developed by the Foundation for Metallurgical Research, MEFOS in Luleå, Sweden. MEFCON has a carbon content hitting rate of $95 \%$ in a window of width $0.007-0.012 \%$ depending on the final carbon content.

To obtain higher estimation accuracy and robustness against modeling errors and measurement noise, it is necessary to utilize multiple information sources, i.e. both a model and the relevant measurements. This calls for feedback of the estimation error, i.e. constructing an observer. Since the process is highly nonlinear and has direct coupling between input and and output, this is a nontrivial task.

Observers for nonlinear systems are for example treated in [2]. The following approaches are often applied for observer design.

Stochastic methods Utilizing the statistical properties of the disturbances, the Kalman filter gives optimal estimates of the state for a linear system. The Extended Kalman Filter and the Statis-

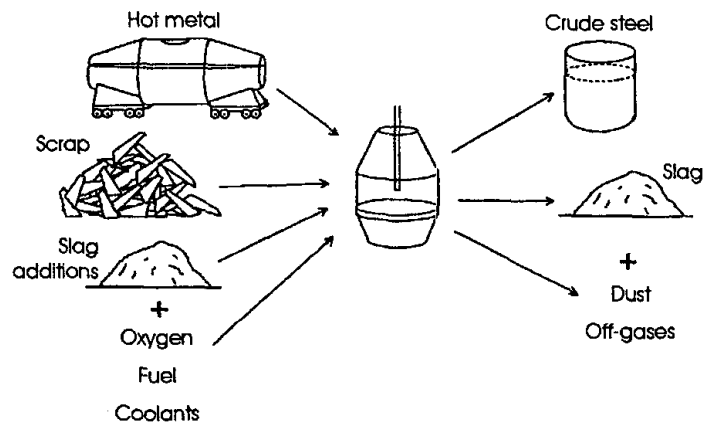

Fig. 1. The top blown converter process

tically Linearized Filter are methods which seek to extend this property to observers for nonlinear systems.

Linearization It is sometimes possible to find a transformation that brings the nonlinear system into a linear one. If this global linearization fails, it is still often possible to perform a local linearization, i.e. to approximate the nonlinear system by a linear system in the vicinity of operating points. Either way there are numerous design techniques for linear systems.

Gain scheduling (for example the Extended Linearization Method) gives desired behaviour (in terms of $e$.g. eigenvalues or convergence rate) in the neighborhood of operating points. Uncertainty approach By regarding the nonlinearities as perturbations from a linear system, methods for observer design for uncertain linear systems can be used.

\section{PROCESS DESCRIPTION}

Fig. 1 is a schematic drawing of the converter process with its raw materials and products.

The operation is started by charging scrap and hot metal into the vessel. Also slag forming agents are fed into the converter. Other additives, such as fuel, in the form of ferrosilicon or coke, and cooling agents, in the form of iron oxides, can be added throughout the blow.

Oxygen $\left(\mathrm{O}_{2}\right)$ is blown at a supersonic speed onto the metal surface and oxidises the metal components, mainly iron $(\mathrm{Fe})$, silicon (Si), manganese $(\mathrm{Mn})$, and carbon $(\mathrm{C})$. The oxides, together with metal droplets, form a foaming slag, in which more carbon will react with the oxides and produce carbon monoxide 
(CO). In combination with oxygen, some of the carbon monoxide will produce carbon dioxide $\left(\mathrm{CO}_{2}\right)$.

The process is controlled by an operator, who judge the state of the process based upon a number of measurements, for instance sound level measurement obtained by a sonicmeter and analysis of the off-gas [3]. The sound level gives information of the thickness of the foaming slag, while the off-gas analysis provides information on the decarburization rate, i.e. the rate at which carbon is oxidised. Other commonly used measurements are off-gas temperature and lance vibration. It is also possible to obtain measurements of temperature and oxygen level in the liquid metal using a sublance or an expendable drop sensor. The sublance also gives information of the carbon content, but these measurements can only be done at discrete times, typically once every blow.

The process is stopped by the operator when the content of carbon in the metal is considered right, based upon the available measurements. The operator may also visually monitor the flame drop above the converter mouth. The quality of the final product is therefore highly dependent on the experience and the judgement of the individual operator.

\section{PROCESS MODEL OF THE STEEL CONVERTER}

A process model (Fig. 2) has been developed at the Division of Metallurgy of the Royal Institute of Technology in Stockholm.

The principles behind the model can be described as follows, referring to the blocks of the figure.

$C B A L$, SIBAL Integrators keeping track of the carbon and silicon content, respectively.

STOFT Calculates the generation of dust, which is assumed to be $10 \mathrm{~kg} / \mathrm{ton}$ of steel.

ANALYS Converting the total contents (in moles) into fractions. $D E K O L 1$ For the main part of the blow, the blowing rate of oxygen will be the rate limiting step for the decarburization, with an initially strong influence from the Si oxidation. At low carbon content the decarburization rate becomes diffusion controlled and thus proportional to the difference between the actual and the lowest achievable carbon content.

GASCOMP This module relates the analysis of the off-gases to the decarburization rate. It is assumed that oxygen from air that leaks in reacts with the carbon monoxide until there is no $\mathrm{CO}$ in the off gases leaving the converter. $\mathrm{O}_{2}$ will be present in the off gases until the $\mathrm{CO}$ amount in the off gases exceeds twice the amount of $\mathrm{O}_{2}$ in the leakage air.

Expressing the above in mathematical terms yields the differential equation

$$
\begin{aligned}
\dot{p} & =A p+B w+E h\left(p, f_{\mathrm{O}_{2}}\right) \\
\psi & =g\left(h\left(p, f_{\mathrm{O}_{2}}\right), f_{\mathrm{og}}\right)
\end{aligned}
$$

where the state vector $p=\left[\begin{array}{ll}p_{\mathrm{Si}} & p_{\mathrm{C}}\end{array}\right]^{T}$ represents the total contents of $\mathrm{C}$ and $\mathrm{Si}$ in the metal, given in moles. See Fig. 2 for notation.

The metal analysis $\rho$ is related to the state vector as

$$
\rho=\left[\begin{array}{ll}
\rho_{\mathrm{Si}} & \rho_{\mathrm{C}}
\end{array}\right]^{T}=\frac{1}{m}\left[\begin{array}{ll}
K_{\mathrm{Si}} p_{\mathrm{Si}} & K_{\mathrm{C}} p_{\mathrm{C}}
\end{array}\right]
$$

where $K_{\mathrm{Si}}$ and $K_{\mathrm{C}}$ represent the molar weight of silicon and carbon, respectively, and $m$ is the total weight of the liquid metal, i.e.

$$
m=K_{\mathrm{Fe}} p_{\mathrm{Fe}}+K_{\mathrm{Si}} p_{\mathrm{Si}}+K_{\mathrm{C}} p_{\mathrm{C}}
$$

where $K_{\mathrm{Fe}}$ and $p_{\mathrm{Fe}}$ are the molar weight and content of iron in the liquid metal.

The mass of the liquid metal is dominated by the iron component which varies only slightly due to oxidation and adding of scrap. Considering the mass as constant is thus motivated and simplifies the model significantly.

Oxidation of $\mathrm{Si}$ and forming of dust of $\mathrm{C}$ and $\mathrm{Si}$ is assumed to be proportional to the contents of these components. Thus the linear part of (1) is asymptotically stable since

$$
A=\left[\begin{array}{cc}
-\left(k_{\theta}+k_{S i}\right) & 0 \\
0 & -k_{\theta}
\end{array}\right]
$$

where the coefficients $k_{\theta}$ and $k_{S i}$ are both positive.

The function $h\left(p, f_{\mathrm{O}_{2}}\right)$ denotes the decarburization rate and therefore only affects the content of $\mathrm{C}$ through the vector $E=$ $\left[\begin{array}{ll}0 & -1\end{array}\right]^{T}$.

\section{A. Input signals}

Inflow of oxygen and the off-gas flow are represented by $f_{\mathrm{O}_{2}}$ and $f_{\text {og }}$, respectively. The contribution of $\mathrm{C}$ and $\mathrm{Si}$ from scrap and other additives constitute the vector $w$. It is assumed that the additives dissolve at a constant rate and thus the elements of $w$ have the form

$$
w_{i}(t)= \begin{cases}m_{i} / T_{i} & t_{i}<t<t_{i}+T_{i} \\ 0 & \text { Otherwise }\end{cases}
$$

where $t_{i}$ is the time at which the amount $m_{i}$ of $\mathrm{C}$ or Si from additive $i$ is added and $T_{i}$ is the time it takes for the additive to completely dissolve. The matrix $B$ consists of two rows of zeros and ones which determines whether the corresponding additive contributes to the amount of $\mathrm{C}$ or $\mathrm{Si}$.

The main source of uncertainty in the model arises here. Both the dissolving times $T_{i}$ and the amounts $m_{i}$ of $\mathrm{C}$ and $\mathrm{Si}$ in particularly the scrap are poorly known.

\section{B. Output signals}

The measured output vector $\psi$ (see Appendix A) is the off-gas analysis. It has a time delay due to the time it takes to transport the gas to the analysis equipment but this is neglected in the analysis.

Since the three components in the off-gas analysis depend only on the decarburization rate, they can be regarded as redundant measurements of the latter. An estimate $\bar{y}$ of the decarburization rate can therefore be obtained from the analysis using e.g. a least squares criterion.

$$
\vec{y}=\phi\left(\psi, f_{\mathrm{og}}\right)
$$

By using this estimate as output signal, it is possible to reduce the nonlinear multiple output system (1) into a single output system with a nonlinear output transformation and output injection.

$$
\begin{aligned}
\dot{p} & =A p+B w+E y \\
y & =h\left(p, f \mathrm{O}_{2}\right)
\end{aligned}
$$




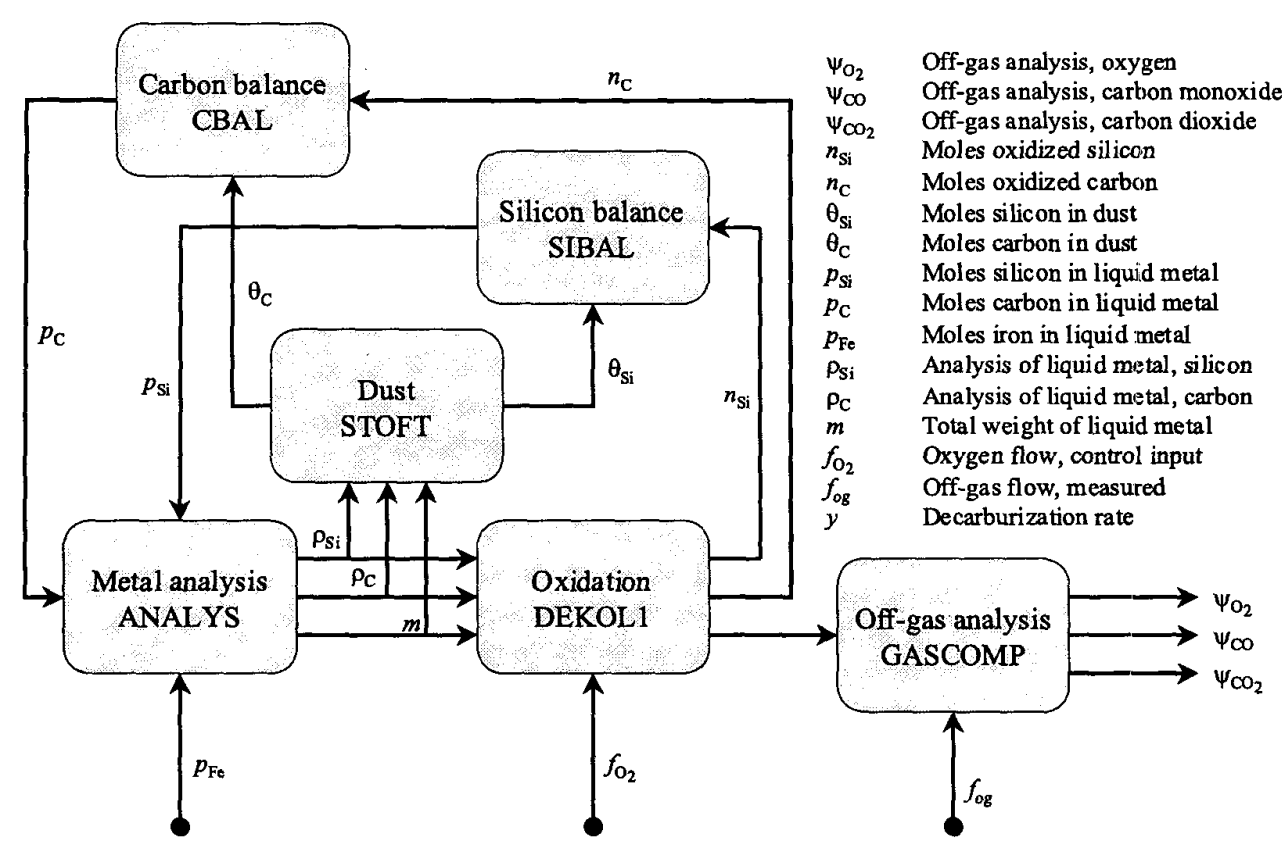

Fig. 2. The process model

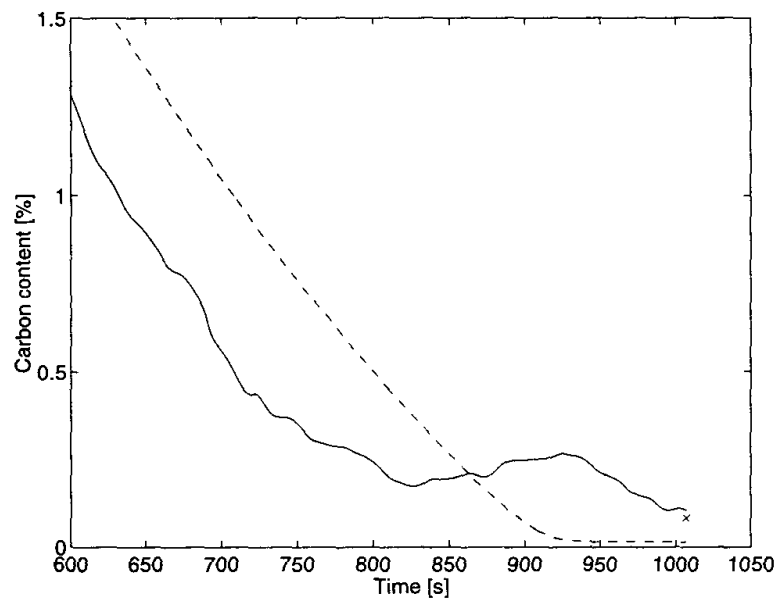

Fig. 3. Estimate of carbon content from experimental data using no feedback (dashed line) and linear feedback (solid line). Cross marks measured final value

When the model is run in open-loop (Fig. 3, dashed line), it does not produce estimates of the carbon content that agree with the measured final values (Fig. 3, cross). Feedback of the off-gas analysis, however, has the potential to improve the results.

\section{STATE ESTIMATION}

A solution where all three components of the off-gas analysis are fed back to the model (solution $A$ in Fig. 4) requires a complicated nonlinear feedback transformation. If the analysis is used to produce an estimate of the decarburization rate, however, a simpler feedback can be used (solution B).

A linear feedback can be adjusted so that the estimated carbon content converges to the measured final value (Fig. 3, solid line). This is however accomplished at the price of unreliable estimates during the rest of the blowing period. Note for example that the carbon content seems to increase during one time interval which is not realistic.

The failure of the linear feedback can be explained with the sensitivity of the output with respect to the state vector (Appendix $\mathrm{A}$ ), i.e.

$$
h^{\prime}\left(p, f_{\mathrm{O}_{2}}\right) \triangleq \frac{\partial h\left(p, f_{\mathrm{O}_{2}}\right)}{\partial p}
$$

Fig. 5 shows the sensitivity to the silicon content (dotted) and to the carbon content (dash-dotted). Since the output signal is expressed in moles per second and the unit of the state vector is moles, the sensitivity has the unit $s^{-1}$. It can be seen that the output is sensitive to the carbon content only during the last part of the blowing period. Intuitively, there is little gain in feeding back the output error to a particular state variable when the output is insensitive to that state variable.

An observer is therefore proposed where the output error is weighted by the sensitivity of the output with respect to the state i.e.

$$
\begin{aligned}
& \dot{\hat{p}}=A \hat{p}+B w+E y+k h^{\prime}\left(\hat{p}, f_{\mathrm{O}_{2}}\right)(\bar{y}-\hat{y}) \\
& \hat{y}=h\left(\hat{p}, f_{\mathrm{O}_{2}}\right)
\end{aligned}
$$

where the gain $k$ is a scalar.

The suggested observer could be compared to the Extended 

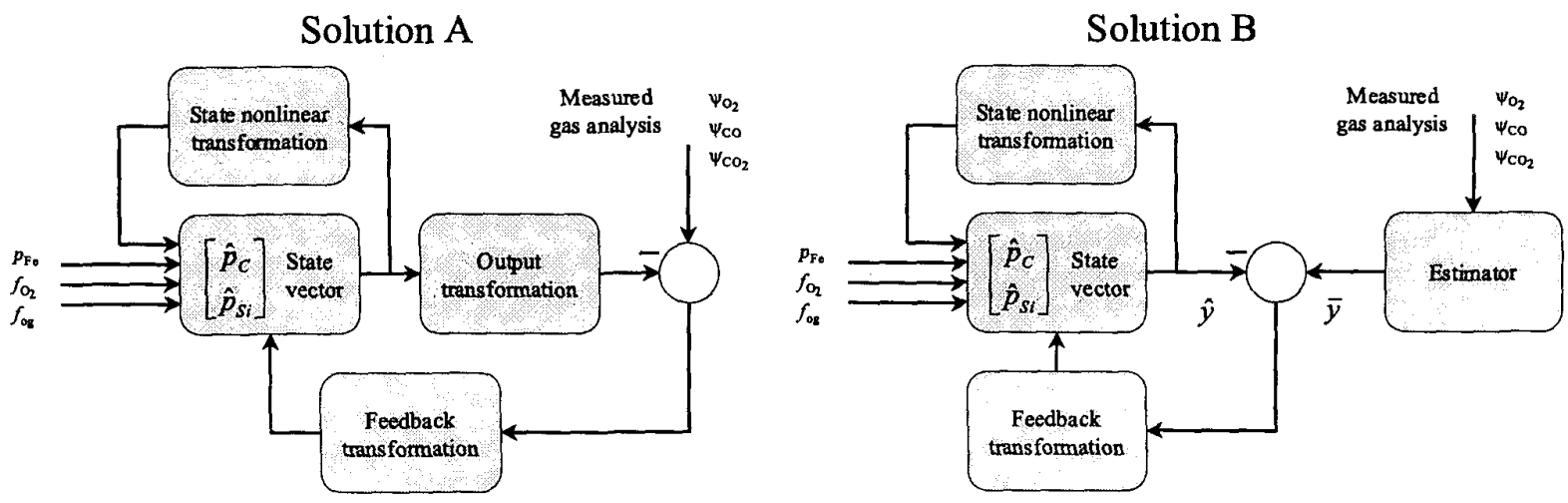

Fig. 4. Two observer structures.

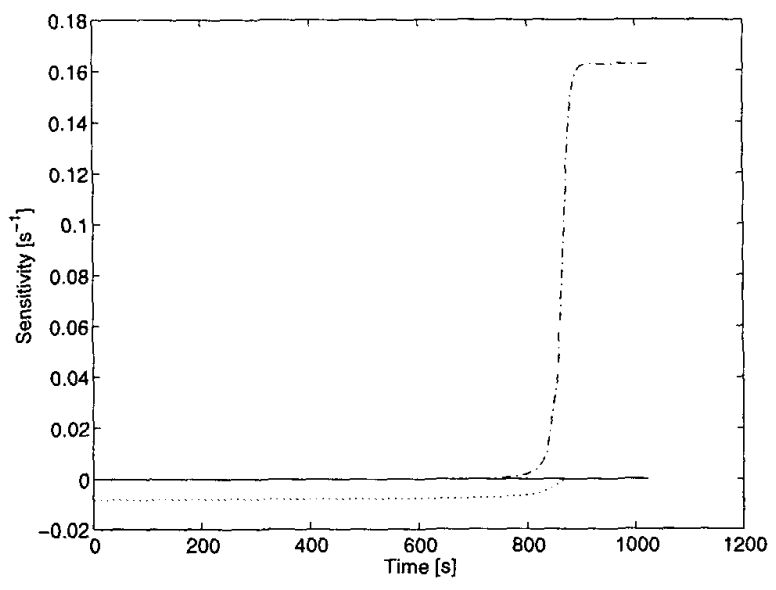

Fig. 5. Sensitivity of the output to the silicon content (dotted) and to the carbon content (dash-dotted) during an entire blow

Kalman Filter where the output error is fed back using the gain

$$
K(t)=P(t) h^{\prime}\left(\hat{p}, f_{\mathrm{O}_{2}}\right) R^{-1}(t)
$$

where $R(t)$ represents the covariance of the measurement noise and $P(t)$ is an approximation of the covariance of the estimation error. The latter is obtained by solving a time-varying Riccati equation, which leads to considerable computational complexity and makes it difficult to verify the stability of the observer.

Analogies can also be made to the MIT rule for updating parameters of an adaptive control system [4]. The parameters $\theta$ are changed along the negative gradient of a quadratic loss function which leads to

$$
\dot{\theta}=-\gamma e \frac{\partial e}{\partial \theta}
$$

where $e$ is the output error and $\gamma$ is a weighting factor. Identifying $\hat{p}$ as the parameter vector and $\bar{y}-\hat{y}$ as the output error this leads to

$$
\dot{\hat{p}}=-\gamma(\bar{y}-\hat{y}) \frac{\partial}{\partial \hat{p}}(\bar{y}-\hat{y})=\gamma h^{\prime}\left(\hat{p}, f_{\mathrm{O}_{2}}\right)(\bar{y}-\hat{y})
$$

which is the feedback term of (3).

\section{A. Stability analysis}

Proposition 1: Given system (2) and observer (3), then the error $\tilde{p}=p-\hat{p}$ is locally asymptotically stable in the neighborhood of $\tilde{p}=0$ if $k \geq 0$ and $A+A^{T}$ is negative definite.

Proof. The error dynamics are

$$
\dot{\tilde{p}}=A \tilde{p}-k h^{\prime}\left(\hat{p}, f_{\mathrm{O}_{2}}\right)(\bar{y}-\hat{y})
$$

Linearizing (4) around $\tilde{p}=0$ yields

$$
\dot{\tilde{p}} \approx \Phi \tilde{p}
$$

where

$$
\begin{aligned}
\Phi & =\left.\frac{\partial \dot{\tilde{p}}}{\partial \tilde{p}}\right|_{\tilde{p}=0} \\
& =A-\left.k h^{\prime}\left(\hat{p}, f_{\mathrm{O}_{2}}\right) h^{\prime T}\left(\tilde{p}+\hat{p}, f_{\mathrm{O}_{2}}\right)\right|_{\tilde{p}=0} \\
& =A-k h^{\prime} h^{\prime T}
\end{aligned}
$$

Assume the Lyapunov function

$$
V=\tilde{p}^{T} \tilde{p}
$$

which is positive definite. Then

$$
\dot{V}=\dot{\tilde{p}}^{T} \tilde{p}+\tilde{p}^{T} \dot{\tilde{p}}=\tilde{p}^{T}\left(\Phi+\Phi^{T}\right) \tilde{p}
$$

The linearized error system (5) is asymptotically stable if the Lyapunov function $V$ is positive definite and its time derivative $\dot{V}$ is negative definite [5]. Showing stability thus reduces to showing that $\Phi+\Phi^{T}=A+A^{T}-2 k h^{\prime} h^{T}$ is negative definite. The largest eigenvalue of $\Phi+\Phi^{T}$ is

$$
\begin{aligned}
\lambda_{\max }\left(\Phi+\Phi^{T}\right) & =\lambda_{\max }\left(A+A^{T}-2 k h^{\prime} h^{\prime T}\right) \\
& \leq \lambda_{\max }\left(A+A^{T}\right)+\lambda_{\max }\left(-2 k h^{\prime} h^{\prime T}\right) \\
& =\lambda_{\max }\left(A+A^{T}\right)-2 k \lambda_{\min }\left(h^{\prime} h^{\prime T}\right)
\end{aligned}
$$

where the inequality is true since $A+A^{T}$ and $-2 k h^{\prime} h^{\prime T}$ are both symmetric [6]. The nonzero eigenvalues of $h^{\prime} h^{\prime T}$ are the 

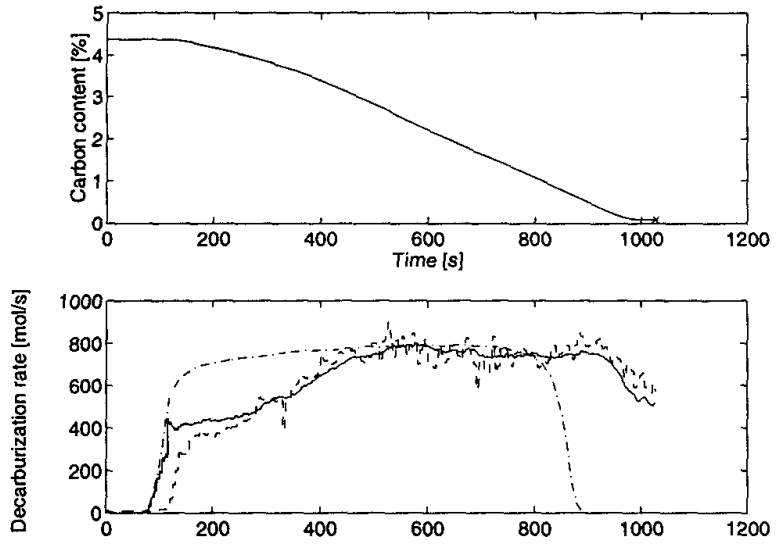

Fig. 6. Estimated decarburization rate. Open loop (dash-dotted line), observer (solid line) and off-gas analysis (dashed line). The upper part shows the corresponding estimated carbon analysis for reference

same as for $h^{\prime T} h^{\prime}$ [6]. But $h^{\prime T} h^{\prime}$ is scalar and its eigenvalue is $h^{\prime T} h^{\prime} \geq 0$ and therefore the minimum eigenvalue of $h^{\prime} h^{\prime T}$ is zero.

Thus

$$
\lambda_{\max }\left(\Phi+\Phi^{T}\right) \leq \lambda_{\max }\left(A+A^{T}\right)
$$

which is negative since $A+A^{T}$ is negative definite. Since the largest eigenvalue of $\Phi+\Phi^{T}$ is negative, $\Phi+\Phi^{T}$ must be negative definite and the proposition follows.

The condition on $A+A^{T}$ in proposition 1 is fulfilled for the converter model since $A$ is diagonal with negative diagonal elements.

\section{B. Experimental results}

The output of the observer is an estimate of the decarburization rate (lower part of Fig. 6 solid line) which is very similar to the estimate obtained from the off-gas analysis (dashed line), but lacking the large disturbances of the latter. The estimate produced by the open-loop simulation (dash-dotted line) is also included in the figure for comparison. The reason to the large deviation from the measured decarburization rate at the beginning of the blow, when run in open loop, is that the model does not take the initial Fe oxidation into account. The decarburization rate will therefore be too high initially. This leads to a too early depletion of carbon and the result will thus be a too low estimation of the final carbon content.

Fig. 7 (upper part) shows the estimated carbon content using the proposed observer with the gain $k=350$. It can be seen that the observer estimate converges to the measured final value of the carbon content (cross). The lower part of the figure shows the corresponding estimates of the decarburization rate from the off-gas analysis (dashed line) and from the observer (solid line).

Note how the estimated carbon analysis seems to follow a straight line until it reaches $\sim 0.2 \%$. This is because the output signal contains very little information about the carbon analysis up to this time, and therefore the estimate is done almost open loop. As the estimated carbon analysis drops, the feedback of

\begin{tabular}{|c|c|c|}
\hline \multicolumn{2}{|c|}{ Carbon content [\%] } & \multirow[b]{2}{*}{ Error $[\%]$} \\
\hline Measured & Estimated & \\
\hline 0.0830 & $0.08 \overline{34}$ & $\overline{0.0004}$ \\
\hline 0.0430 & 0.0418 & -0.0012 \\
\hline 0.0330 & 0.0344 & 0.0014 \\
\hline 0.0260 & 0.0229 & -0.0031 \\
\hline
\end{tabular}

TABLE I

COMPARISON BETWEEN MEASURED AND ESTIMATED CARBON CONTENTS

the off-gas analysis starts to affect the estimate and lead it towards the correct final value.

Table I compares the measured and the final value of the estimated carbon analyses in Fig. 7. Note however that the accuracy of the measurement is limited to three decimals and therefore the values are not quite comparable.

\section{Conclusions}

It has been shown that by utilizing an analytical model and suitable feedback of the off-gas analysis, it is possible to obtain accurate estimates of the metal analysis in a top blown converter process. The dynamics of the error when applying this feedback has been shown to be asymptotically stable.

\section{ACKNOWLEDGEMENTS}

The authors want to thank the personnel of SSAB Oxelösund for supplying the data. Financial support provided by the Swedish National Board for Industrial and Technical Development, NUTEK, is also gratefully acknowledged.

\section{REFERENCES}

[1] http://www.mefos.se/mefcon.htm.

[2] E. A. Misawa and J. K. Hedrick, "Nonlinear observers - a state-of-the-art survey," Journal of Dynamic Systems, Measurment and Control, vol. 111 , pp. 344-352, September 1989.

[3] D. Widlund, A. Medvedev, and R. Gyllenram, "Towards model-based closed-loop control of the basic oxygen steelmaking process," in Preprints of the 9th IFAC Symposium Automation in Mining, Mineral and Metal Processing 1998, 1998.

[4] K.-J. Âström and B. Wittenmark, Adaptive Control. Addison-Wesley Publishing Company, Inc., 2 ed., 1995.

[5] J.-J. E. Slotine and W. Li, Applied Nonlinear Control. Prentice Hall, 1991.

[6] F. Zhang, Matrix Theory. Basic Results and Techniques. Springer-Verlag, 1999.

\section{APPENDIX}

\section{OUTPUT TRANSFORMATION}

The off-gas analysis is modeled as

$$
\begin{aligned}
\psi & =\left[\begin{array}{lll}
\psi_{\mathrm{CO}_{2}} & \psi_{\mathrm{CO}} & \psi_{\mathrm{O}_{2}}
\end{array}\right]^{T} \\
& =g\left(h\left(p, f_{\mathrm{O}_{2}}\right), f_{\mathrm{og}}\right)=\gamma(\eta) \\
& = \begin{cases}\gamma_{1}(\eta) & \eta<\frac{2 \alpha}{1+2 \alpha} \\
\gamma_{2}(\eta) & \eta \geq \frac{2 \alpha}{1+2 \alpha}\end{cases}
\end{aligned}
$$

where

$$
\begin{aligned}
& \gamma_{1}(\eta)=\frac{1}{2-\eta}\left[\begin{array}{lll}
2 \eta & 0 & 2 \alpha(1-\eta)-\eta
\end{array}\right]^{T} \\
& \gamma_{2}(\eta)=\frac{1}{1-\alpha+\alpha \eta}\left[\begin{array}{lll}
2 \alpha-2 \alpha \eta & 2 \alpha(\eta-1)+\eta & 0
\end{array}\right]^{T}
\end{aligned}
$$



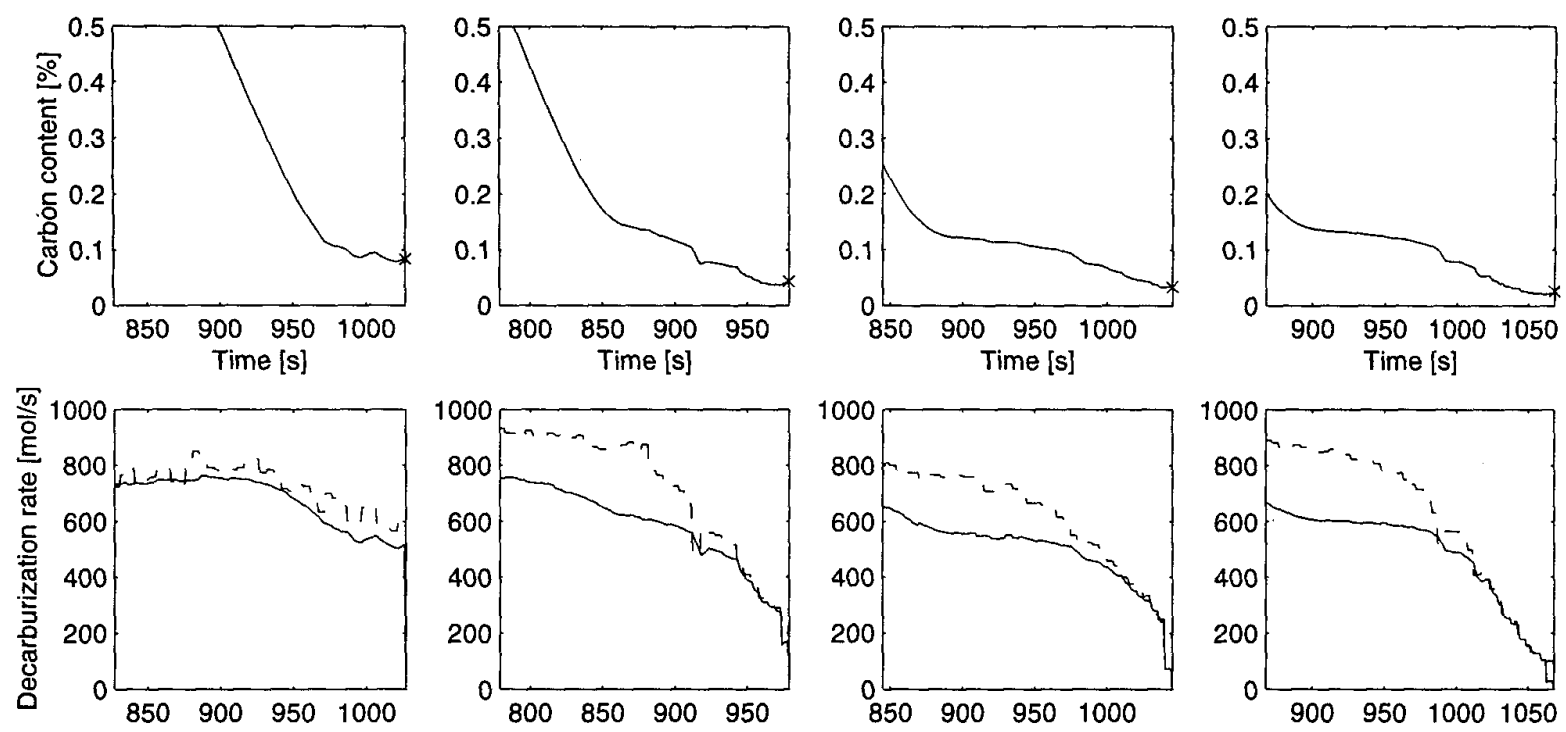

Fig. 7. Estimated carbon content and decarburization rate for four heats using measured data. Measured decarburization rate (dashed lines), observer estimate (solid lines) and measured final value of carbon content (crosses).

and

$$
\eta=\beta \frac{h\left(p, f_{\mathrm{O}_{2}}\right)}{f_{\mathrm{og}}}
$$

is the mole fraction of carbon in the off-gas, in $\mathrm{mol} / \mathrm{m}^{3}$. The constant $\alpha$ represents the fraction of oxygen in air while $\beta$ is the amount of molecules (in moles) in an ideal gas at normal pressure and temperature. The two branches in (6) represent the cases when there is surplus oxygen and carbon monoxide in the off-gases, respectively.

The decarburization rate is modeled as

$$
h\left(p, f_{\mathrm{O}_{2}}\right)=\frac{1}{\frac{1}{v_{1}}+\frac{1}{v_{2}}}
$$

where

$$
\begin{aligned}
& v_{1}=\kappa_{\mathrm{C}}\left(\rho_{\mathrm{C}}-\rho_{\mathrm{C}}^{0}\right)=\kappa_{\mathrm{C}}\left(\frac{K_{\mathrm{C}} p_{\mathrm{C}}}{m}-\rho_{\mathrm{C}}^{0}\right) \\
& v_{2}=2\left(f_{\mathrm{O}_{2}}-k_{\mathrm{Si}} p_{\mathrm{Si}}\right)
\end{aligned}
$$

where $\rho_{\mathrm{C}}^{0}$ is the lowest achievable carbon content.

The sensitivity of the output with respect to the state is

$$
\begin{aligned}
\frac{\partial h\left(p, f_{\mathrm{O}_{2}}\right)}{\partial p} & =\left[\begin{array}{ll}
\frac{\partial h\left(p, f_{\mathrm{O}_{2}}\right)}{\partial p_{\mathrm{Si}}} & \frac{\partial h\left(p, f_{\mathrm{O}_{2}}\right)}{\partial p_{\mathrm{C}}}
\end{array}\right]^{T} \\
& =\left[\begin{array}{ll}
-\frac{2 k_{\mathrm{Si}} v_{1}^{2}}{\left(v_{1}+v_{2}\right)^{2}} & \frac{\kappa_{\mathrm{C}} K_{\mathrm{C}} v_{2}^{2}}{m\left(v_{1}+v_{2}\right)^{2}}
\end{array}\right]^{T}
\end{aligned}
$$

